\title{
Stage IB Esophageal Adenocarcinoma AJCC v7
}

National Cancer Institute

\section{Source}

National Cancer Institute. Stage IB Esophageal Adenocarcinoma A/CC V7. NCI

Thesaurus. Code C87783.

Stage IB includes: (T1, N0, M0, G3); (T2, N0, M0, G1-2, GX). T1: T umor invades lamina propria, muscularis mucosae, or submucosa. T2: Tumor invades muscularis propria. N0: No regional lymph node metastasis. M0: No distant metastasis. G1: Well differentiated. G2: Moderately differentiated. G3: Poorly differentiated. GX: Grade cannot be assessedstage grouping as G1. (AJCC 7th ed.) 